\title{
Steam generation unit in a simple version of biomass based small cogeneration unit
}

\author{
Krzysztof Sornek $^{1, a}$, Mariusz Filipowicz ${ }^{1}$, Mateusz Szubel ${ }^{1}$, Estera Bożek ${ }^{1}$, Krzysztof Izdebski ${ }^{1}$ \\ ${ }^{1}$ AGH University, Faculty of Energy and Fuels, Department of Sustainable Energy Development
}

\begin{abstract}
The organic Rankine cycle (ORC) is a very promising process for the conversion of low or medium temperature heat to electricity in small and micro scale biomass powered systems. Classic ORC is analogous to Clausius-Rankine cycle in a steam power plant, but instead of water it uses low boiling, organic working fluids. Seeking energy and economical optimization of biomass-based ORC systems, we have proposed some modifications e.g. in low boiling fluid circuit construction. Due to the fact that the operation of a micro steam turbine is rather inefficient from the technical and economic point of view, a specially modified air compressor can be used as a steam piston engine. Such engine should be designed to work at low pressure of the working medium. Studies regarding the first version of the prototype installation were focused on the confirmation of applicability of a straw boiler in the prototype ORC power system. The results of the previous studies and the studies described in the paper (on the new cogeneration unit) confirmed the high potential of the developed solution. Of course, many further studies have to be carried out.
\end{abstract}

\section{Introduction}

According to the EU Energy, Transport and GHG Emissions Trends to 2050, the overall share of renewable energy in electricity production will reach $50 \%$ in 2050 . This is associated with the expected dynamic development of distributed energy systems - especially with the use of micro and small scale combined heat and power generation units (CHP) [1].

Potentially, the most promising energy source capable to largely meet the local heat and electricity demand is solid biomass (e.g. wood, straw and pellet). The available heat capacity, however, makes these kinds of fuels impossible to be converted to electrical power efficiently with the use of conventional power generation methods. In this case, modern appliances based on biomass combustion and dedicated to use in energy systems of individual houses and entire settlements, will be based on the use of the Organic Rankine Cycle (ORC) operation principles. Evaporation of the working fluid (the steam of which drives a turbine coupled with an electricity generator), will occur as a result of high temperature generated in biomass boilers. Heat transfer from the biomass boiler to the working fluid will be realized with the use of a hot oil circuit, with a heat exchanger placed inside the boiler.

Due to many technical problems relating to the state of the art, it is necessary to seek appropriate methods of high efficiency electricity generation in biomass-based appliances.

\footnotetext{
a Corresponding author: krzysztof.sornek@agh.edu.pl
}

\section{Organic Rankine Cycle (ORC)}

The Organic Rankine Cycle (ORC) is a process for conversion of low or medium temperature heat to electricity. The classical ORC plant is analogous to the Clausius-Rankine steam power plant, but instead of water it uses low boiling, organic working fluids. The use of organic working fluids results in the ORC cycle having several advantages over the steam cycle. Higher molecular weight than water increases the mass flow rate of the fluid for the same size of turbine. The result is a better power generation efficiency (about 10-20\%). Most importantly, the boiling point of ORC fluids is much lower than that of water, so they can be applied in lower temperatures. The classical ORC scheme is shown in Figure 1.

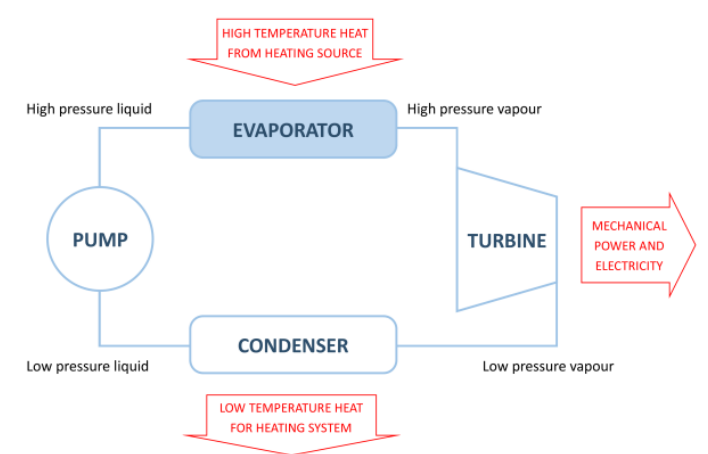

Figure 1. The idea of a small cogeneration system. 
The classical ORC system has some disadvantages. The greatest difficulty is related to the micro/small steam turbine construction - its design parameters cannot be transferred directly from the large scale solutions. Moreover, additional works connected with proper cooperation between ORC units and biomass boilers are required. Such works should be focused particularly on the development of the optimal high temperature heat collection system, the adjustment of the boiler's operation parameters to heating and power generation system requirements, as well as be concentrated on meeting the requirements regarding the carbon dioxide emission into the atmosphere.

\section{The prototype of a combined heat and power system with a modified ORC unit}

Due to real demand for combined heat and power systems, construction of a small-scale CHP unit was commenced. The idea was to use the heat from exhaust gases (which are normally released to atmosphere) in electricity generation. This means that heat is still the main product of the combustion process and electricity is only an additional co-product (about 90\% of the generated heat is used directly to meet the heating needs).

Our prototype unit is based on the biomass (especially straw) boiler and is dedicated e.g. to the use in farms, settlements etc. The boiler has a heat capacity of $180 \mathrm{~kW}$ and a special counter flow combustion system with two combustion chambers. In the first big chamber the fuel is mainly gasified and then afterburned in the second small chamber. Based on knowledge concerning the temperature distribution in the boiler (many studies were previously conducted using a special measurement and control system, based on a PLC controller), a high temperature heat collection system was developed [2]. This system is equipped with a special oil heat exchanger, which receives heat from the exhaust gases and transports it via the oil circuit to the low boiling fluid circuit. The oil heat exchanger is made of steel pipes and is placed inside the second combustion chamber (see Figure 2). It is connected with the rest of the system using the pipes led out through the upper door of the boiler.

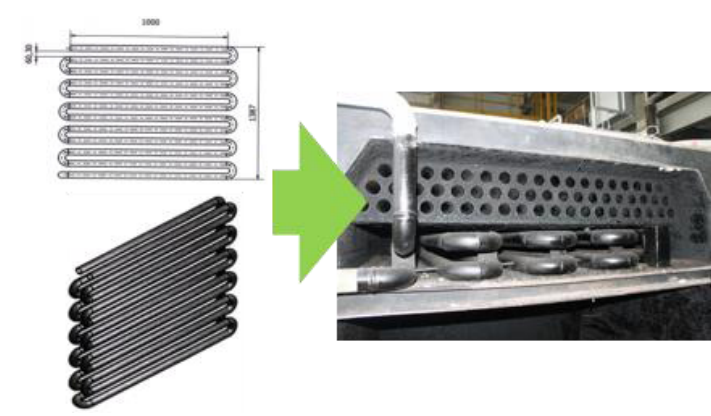

Figure 2. The location of the oil heat exchanger in the second combustion chamber of the boiler.

The oil circuit consists of two shell and tube heat exchangers, connected in such a way that it is possible to use either one or two heat exchangers. In the case of the operation of two heat exchangers, they can be connected in series or in parallel. The oil flow is forced by an oil pump, controlled by a typical frequency inverter. The cold side of the shell and the tube heat exchangers is currently equipped with a first version of a steam cycle circuit, including a small steam engine with mechanical power of about one HP. This temporarily used engine will be shortly replaced by a dedicated steam engine with parameters adapted to the entire system. Due to a big similarity with existing units like combustion engines, air compressors, pneumatic engines, etc. (e.g. cylinders, pistons, crankshafts), the dedicated steam engine will be based on the units referred to above. Most likely, a cylinder with a piston and a crankshaft will be used, the inlet and outlet valves will be removed, additional pipes for steam and flywheel will be added and a cam with a camshaft will be constructed. The design work is in progress. In comparison to steam microturbines (currently in the stage of application), lower efficiency is expected on one hand, but unit production costs will be significantly reduced on the other [3].

The proper choice of the organic working fluid poses a certain challenge. One of the most important aspects is the temperature possible to obtain in the oil heat exchanger, which can range from $100^{\circ} \mathrm{C}$ to about $250^{\circ} \mathrm{C}$. Based on a literature analysis [e.g. 4], R11, R141b, R113 and R123 fluids manifest the best thermodynamic performances, while R245fa and R245ca are the most environmentally-friendly. Finally, the working fluid will, in our case, be chosen based on the final CHP unit parameters - the current studies were conducted with water instead.

\subsection{Diagram of the proposed cogeneration system including the control and measuring system}

The general diagram of the prototype small CHP system, including elements described above, is shown in Figure 3. A monitoring and control system, developed especially for the designed small scale cogeneration system, uses e.g. thermocouples (located in both of the boiler's combustion chambers), resistance temperature sensors (located in the oil and water parts), flow meters (for the measurement of boiler power, as well as oil and water circuits), inverters (connected with the fan and pumps) and an exhaust gas analyser.

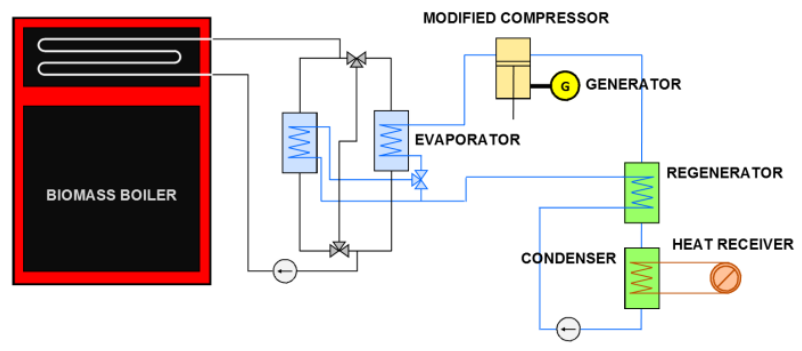

Figure 3. The general diagram of the prototype small CHP system. 
Measurements were made using the modular Wago I/O System with a programmable logic controller (PLC). The control of the system operation is carried out by a special application, developed in CoDeSys software using CFC (Continuous Function Chart) and ST (Structured Text) programming languages. The diagram including the points of temperature, pressure and flow sensors in the oil and water parts is shown in Figure 4.

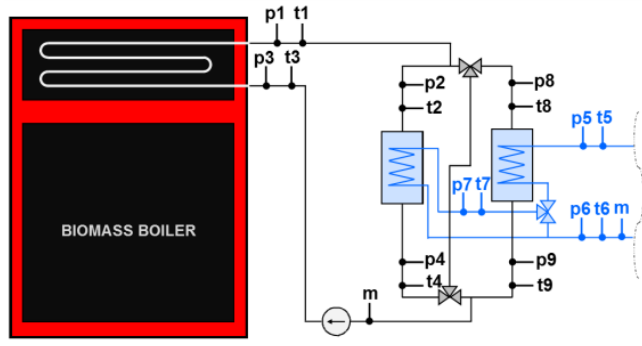

Figure 4. Scheme with marked temperature, pressure and flow sensors in the oil and water parts.

The localization of temperature sensors in the oil circuit is also presented on a visualization shown in Figure 5.

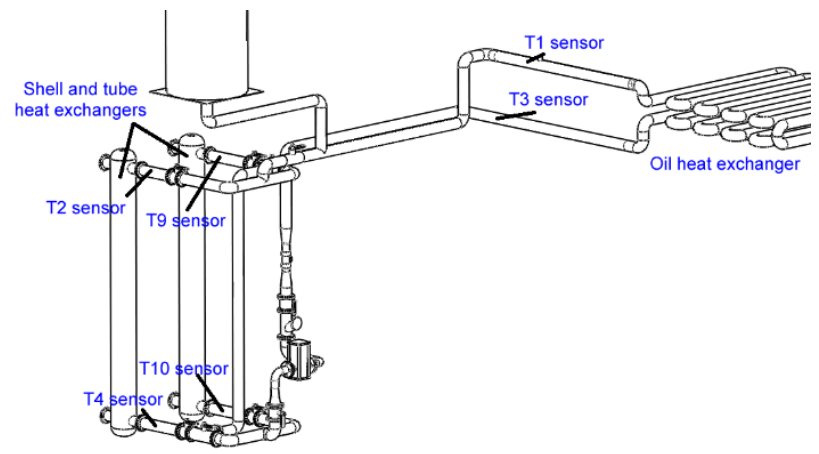

Figure 5. Temperature sensors in the oil part visualization.

\section{Discussion of the results}

The results of previously conducted studies (regarding the start-up of a new cogeneration unit) confirmed the high potential of the developed solution. The following figures illustrate temperature changes in selected points of the oil and water circuits, as well as the temporary capacity of the boiler, oil circuit capacity and water circuit capacity. Studies were conducted for different configurations of shell and tube heat exchangers: single heat exchanger operation, heat exchangers in serial operation and heat exchangers operating in parallel configuration. The control method of the boiler's operation was the same in each case - after 2-3 minutes of combustion process, the air blower was set at $100 \%$ of its capacity and has worked until all fuel has been burnt. The moments of switching on/off the oil pump and the water pump are shown in the figures.

\subsection{The initial configuration with a single shell and tube heat exchanger}

In the initial configuration, only one shell and tube heat exchanger served as an evaporator. During the combustion process, $65 \mathrm{~kg}$ of fuel was burnt, including $60 \mathrm{~kg}$ of straw and $5 \mathrm{~kg}$ of shredded paper. The oil temperature reached a level exceeding $150^{\circ} \mathrm{C}$ (the oil pump started operating at that point). As a result, after 18 minutes of the combustion process, the temporary power in the oil circuit achieved the value of $60 \mathrm{~kW}(1 / 3$ of the nominal heat output of the boiler) and the temporary power in the water part reached the value of $50 \mathrm{~kW}$. The water pump operated at full power since the beginning, so the water temperature reached only about $40^{\circ} \mathrm{C}$. The changes of the oil and cooling water temperature are presented in Figure 6.
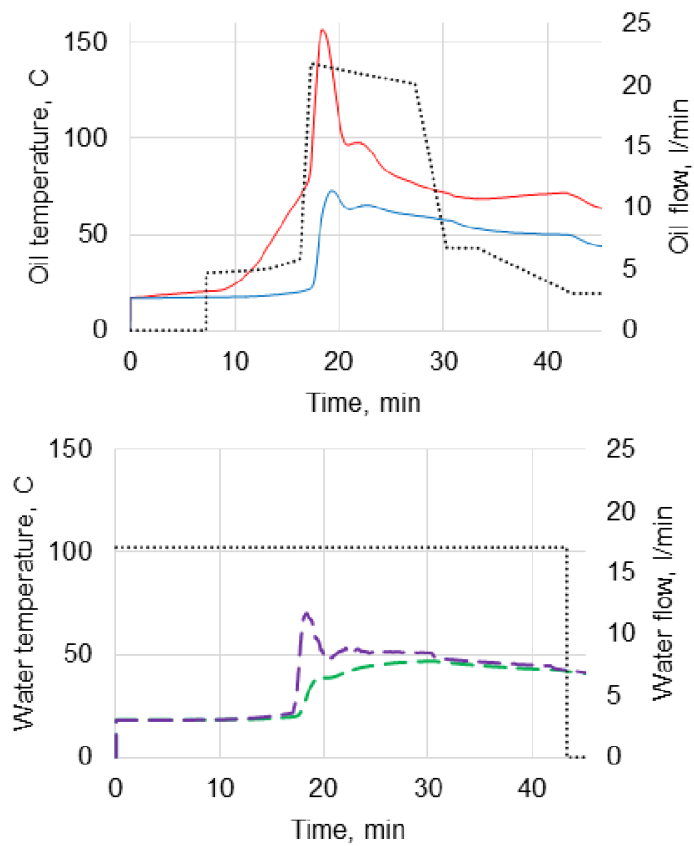

Figure 6. Oil and cooling water temperature changes.

Because of the non-maximum fuel input (six straw bales were burned), the boiler's heating power reached only $\sim 70 \%$ of nominal value. However, this power is not sufficient to provide high and stable power in the oil circuit. Values of the temporary power generated during the combustion process in selected parts of the system are shown in Figure 7.

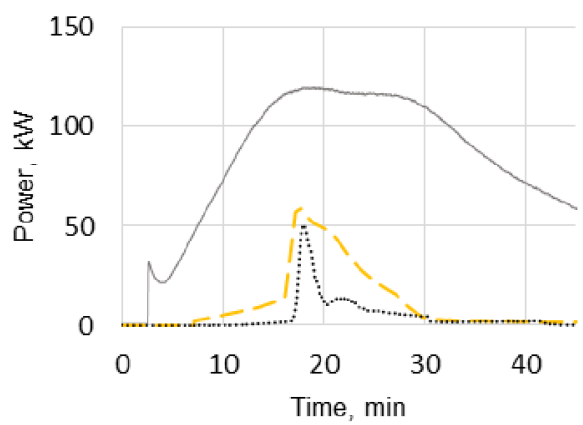

Figure 7. Power values in selected parts of the system. 


\subsection{Shell and tube heat exchangers connected in serial configuration}

In the second configuration, two shell and tube heat exchangers connected in a series were used. Because of the higher flow resistance, significant decrease of the oil flow rate (and therefore the oil mass flow) was visible. It affected the temperature and temporary power changes. Due to the general method of the combustion process (oil circulation pump switched to $100 \%$ power after 5 minutes from start, as well as subsequent inclusion of the water pump), the maximum temperature of the oil and water was achieved in the final phase of the combustion process. Low fuel input, comprised of only $45 \mathrm{~kg}$ of straw (with an average moisture content of $12 \%$ ) and about 5 $\mathrm{kg}$ of shredded paper, caused that the oil temperature at the boiler's outlet to amount to only $110^{\circ} \mathrm{C}$, and the temperature on the return to the boiler did not exceed 50 ${ }^{\circ} \mathrm{C}$ (see Figure 8).
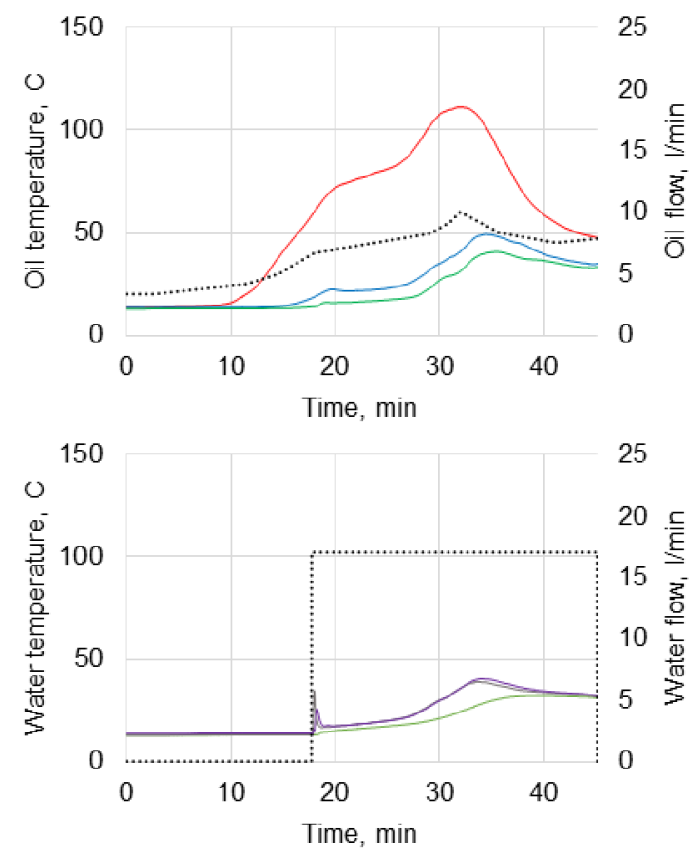

Figure 8. Oil and cooling water temperature changes.

The maximum power in the oil circuit in the case of serial operation of two shell and tube heat exchangers is approximately $25 \mathrm{~kW}$, and the power of the water circuit - about $15 \mathrm{~kW}$ (see Figure 9).

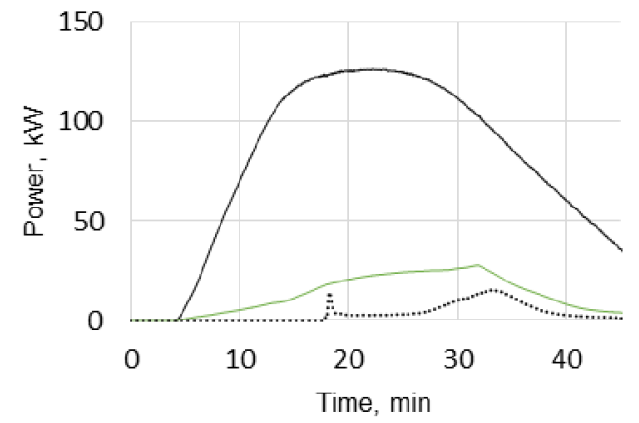

Figure 9. Power values in selected parts of the system.

\subsection{Configuration with shell and tube heat exchangers connected in parallel}

The last configuration consisted in two shell and tube heat exchangers being connected in a parallel. The studies included two combustion processes occurring one by one. The maximum oil temperature reached the level of $120{ }^{\circ} \mathrm{C}$ (at the outlet of the boiler during the second combustion process), while the temperature at the return to the boiler was about $80{ }^{\circ} \mathrm{C}$. Due to the serial connection of the cold sides of the shell and tube heat exchangers, the oil temperature at the outputs was similar. It was noticed that during the second combustion process the oil temperature increased by several percent. The conclusion is that the following combustion processes would cause a higher increase in the temperature - proportionally to the fuel load (see Figure $10)$.
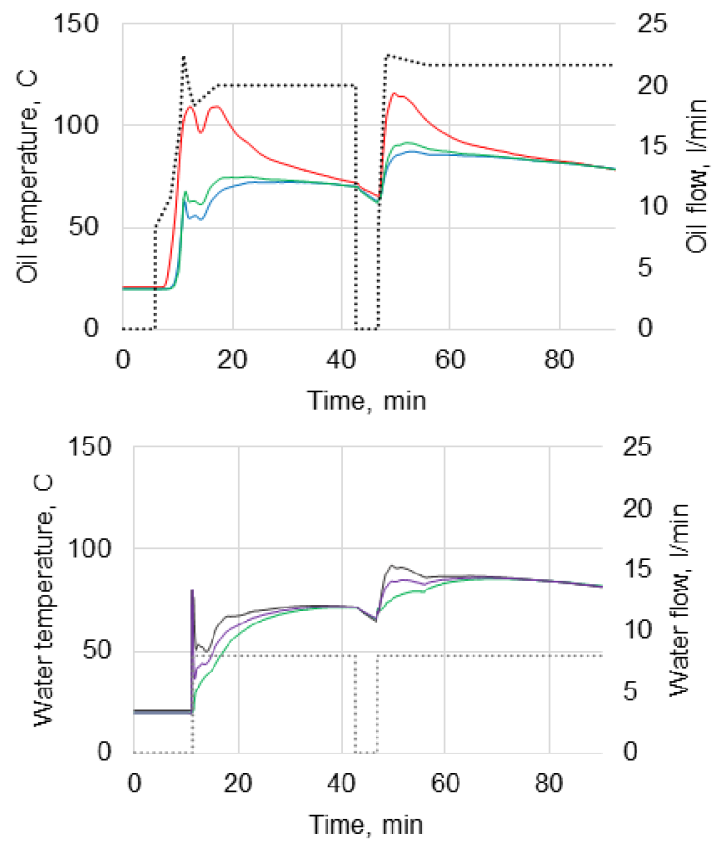

Figure 10. Oil and cooling water temperature changes.

Changes in oil and water temperature are related to power changes in the considered parts of the system. The highest obtained power values in the oil and water circuits were about $45 \mathrm{~kW}$ and $35 \mathrm{~kW}$, respectively. Until now, these values were obtained only temporarily (see Figure 11).

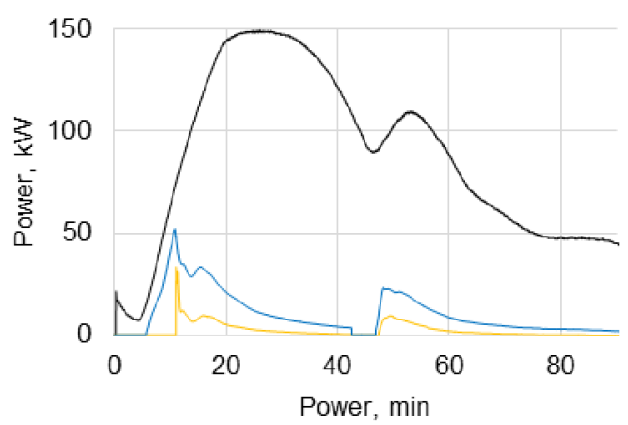

Figure 11. Power values in selected parts of the system. 


\section{Conclusions}

The previously conducted studies show that there are grounds for conducting further research. The expected result is to develop a small prototype cogeneration system based on the use of a straw boiler with an integrated power generation unit.

The results obtained so far display some differences depending on the mode of the oil circuit operation. The highest temporary power was achieved when a single shell and tube heat exchanger was used. On the other hand, in the case of parallel connection of two exchangers, the value of power was most stable during the combustion process. These observations, however, are largely associated with the method of conducting the combustion process and further research is required.

Nevertheless, the power achieved in the oil system exceeded 20 and more percent of the thermal power at some points in time. The expectations relating to the system operation were thus met.

\section{Acknowledgements}

Works conducted under the „Bio-Eco-Matic: Construction of small to medium capacity boilers for clean and efficient combustion of biomass for heating" project.

\section{References}

1. EU Energy, Transport and GHG Emissions Trends to 2050, Reference Scenario, p. 47, http://ec.europa.eu, 2013 [27.04.2014]

2. K. Sornek, M. Tomski, M. Filipowicz, Possibility of the use of biomass in modern, domestic poligeneration systems, Folia Scientiarum Universitatis Technicae Resoviensis No. 283 (2012)

3. K. Sornek, T. Mirowski, E. Bożek, M. Filipowicz, Micro scale multi-generation systems in modern residential buildings, 1 st World multi-conference on Intelligent Building Technologies \& Multimedia Management IBTMM 2013: proceedings of the 7th Congress on Intelligent Building Systems InBus 2013

4. E.H. Wang, H.G. Zhang, B.Y. Fan, M.G. Ouyang, Y. Zhao, Q.H. Mu, Study of working fluid selection of organic Rankine cycle (ORC) for engine waste heat recovery, Energy, Volume 36, Issue 5 (2011) 